\title{
Primary Vitamin D Target Genes of Human Monocytes
}

\author{
Veijo Nurminen ${ }^{1}$, Sabine Seuter ${ }^{2}$ and Carsten Carlberg ${ }^{1 *}$ \\ ${ }^{1}$ School of Medicine, Institute of Biomedicine, University of Eastern Finland, Kuopio, Finland, ${ }^{2}$ Institute for Cardiovascular \\ Physiology, Medical Faculty, Goethe University Frankfurt, Frankfurt, Germany
}

The molecular basis of vitamin $D$ signaling implies that the metabolite $1 \alpha, 25$ dihydroxyvitamin $D_{3}\left(1,25(\mathrm{OH})_{2} \mathrm{D}_{3}\right)$ of the secosteroid vitamin $D_{3}$ activates the transcription factor vitamin $\mathrm{D}$ receptor (VDR), which in turn modulates the expression of hundreds of primary vitamin $D$ target genes. Since the evolutionary role of nuclear receptors, such as VDR, was the regulation of cellular metabolism, the control of calcium metabolism became the primary function of vitamin D and its receptor. Moreover, the nearly ubiquitous expression of VDR enabled vitamin $D$ to acquire additional physiological functions, such as the support of the innate immune system in its defense against microbes. Monocytes and their differentiated phenotypes, macrophages and dendritic cells, are key cell types of the innate immune system. Vitamin D signaling was most comprehensively investigated in THP-1 cells, which are an established model of human monocytes. This includes the $1,25(\mathrm{OH})_{2} \mathrm{D}_{3}$-modulated cistromes of VDR,

Edited by:

Anna M. D. Watson,

Monash University, Australia

Reviewed by:

Behdad Afzali,

National Institute of Diabetes and Digestive and Kidney Diseases (NIDDK), United States

Paul Kenneth Witting,

The University of Sydney, Australia

*Correspondence:

Carsten Carlberg

carsten.carlberg@uef.fi

Specialty section: This article was submitted to Integrative Physiology, a section of the journal

Frontiers in Physiology

Received: 27 November 2018

Accepted: 15 February 2019

Published: 05 March 2019

Citation:

Nurminen $V$, Seuter $S$ and Carlberg C (2019) Primary Vitamin D Target Genes of Human Monocytes. Front. Physiol. 10:194.

doi: 10.3389/fphys.2019.00194 the pioneer transcription factors PU.1 and CEBPA and the chromatin modifier CTCF as well as of the histone markers of promoter and enhancer regions, H3K4me3 and H3K27ac, respectively. These epigenome-wide datasets led to the development of our chromatin model of vitamin $\mathrm{D}$ signaling. This review discusses the mechanistic basis of 189 primary vitamin $\mathrm{D}$ target genes identified by transcriptome-wide analysis of $1,25(\mathrm{OH})_{2} \mathrm{D}_{3}$-stimulated THP-1 cells and relates the epigenomic basis of four different regulatory scenarios to the physiological functions of the respective genes.

\section{Keywords: vitamin D, VDR, epigenome, transcriptome, gene regulation, vitamin D target genes, monocytes}

\section{INTRODUCTION}

In all animal species, in which the cholesterol precursor 7-dehydrocholesterol is exposed to UV-B, the secosteroid vitamin $\mathrm{D}_{3}$ is formed in a non-enzymatic reaction (Hart et al., 2011). At a sunny day with an UV index of 3 or higher humans can produce vitamin $\mathrm{D}_{3}$ in their unprotected skin (Holick, 2011). However, today's lifestyle with predominant indoor activity and textile coverage outdoors as

Abbreviations: $1,25(\mathrm{OH})_{2} \mathrm{D}_{3}, 1 \alpha, 25$-dihydroxyvitamin $\mathrm{D}_{3}$; CAMP, cathelicidin antimicrobial peptide; CD14, CD14 molecule; CEBPA, CCAAT/enhancer binding protein alpha; ChIP-seq, chromatin immunoprecipitation sequencing; CTCF, CCCTC-binding factor; CYP26B1, cytochrome P450 family 26 subfamily B member 1; DR3, direct repeat spaced by 3 nucleotides; FAIRE-seq, formaldehyde-assisted isolation of regulatory elements sequencing; FBP1, fructose-bisphosphatase 1; FC, fold change; IGV, Integrative Genomics Viewer; LD, ligand-dependent; LILRB4, leukocyte immunoglobulin like receptor B4; ORM1, orosomucoid 1; PBMC, peripheral blood mononuclear cells; PU.1, purine-rich box 1; RNA-seq, RNA sequencing; RXR, retinoid X receptor; Spi-1 proto-oncogene (official gene symbol: SPI1); TAD, topologically associated domain; TFE3, transcription factor binding to IGHM enhancer 3; TMEM37, transmembrane protein 37; TSPAN18, tetraspanin 18; TSS, transcription start site; VDR, vitamin D receptor. 
well as seasonal variations in sun intensity at latitudes higher than $30^{\circ} \mathrm{N}$ or lower than $30^{\circ} \mathrm{S}$ often prevents endogenous vitamin $\mathrm{D}_{3}$ synthesis and makes the molecule for a large proportion of the human population an essential micronutrient, i.e., a true vitamin, that needs to be taken up by diet or supplementation with pills (Bendik et al., 2014; Carlberg, 2016). Interestingly, more than 100 years ago a sunshine cure leading to endogenous production of vitamin $\mathrm{D}_{3}$ was used as an efficient therapy of the children's bone malformation disease rickets as well as of the infectious disease tuberculosis (Holick, 1981; Grad, 2004).

Vitamin $\mathrm{D}_{3}$ itself is biologically inert, but hydroxylation at carbon 25 of its side chain into 25-hydroxyvitamin $\mathrm{D}_{3}$ and further hydroxylation at carbon 1 within its A-ring results in the active metabolite $1,25(\mathrm{OH})_{2} \mathrm{D}_{3}$. As a lipophilic molecule $1,25(\mathrm{OH})_{2} \mathrm{D}_{3}$ easily passes through biological membranes and binds with high-affinity $\left(\mathrm{k}_{\mathrm{D}} 0.1 \mathrm{nM}\right)$ to the transcription factor VDR, which is primarily located in the nucleus (Haussler et al., 2013). This explains why vitamin $D_{3}$ has via its metabolite $1,25(\mathrm{OH})_{2} \mathrm{D}_{3}$ major effects on the transcriptome and the resulting proteome of VDR-expressing cell types. Proximal tubule cells of the kidneys are the main production site of the circulating endocrine hormone $1,25(\mathrm{OH})_{2} \mathrm{D}_{3}$, but for para- and autocrine use the molecule is also formed in monocytes, macrophages and dendritic cells of the innate immune system, osteoblasts within bones and keratinocytes of the skin (Hewison, 2012).

When 250-500 million years ago some animal species left the ocean and had to develop a stable calcium-based skeleton, $1,25(\mathrm{OH})_{2} \mathrm{D}_{3}$ developed to a nuclear hormone that, via its receptor, took over the role as main regulator of calcium homeostasis (Bouillon and Suda, 2014). Within the same evolutionary time span, the immune system of vertebrates further evolved and the vitamin $\mathrm{D}$ endocrine system acquired a role in its regulation, in order to mediate a more efficient protection against infectious diseases, such as tuberculosis. Tuberculosis is caused by the intra-cellular bacterium Mycobacterium tuberculosis, the proliferation of which within macrophages is inhibited by vitamin D (Rook et al., 1986). Moreover, after vitamin $\mathrm{D}$ treatment monocytes and macrophages recognize bacterial pathogens more efficiently via pattern-recognition receptors, such as toll-like receptors (Liu et al., 2006). A key player in the anti-microbial effects of $1,25(\mathrm{OH})_{2} \mathrm{D}_{3}$ is the protein cathelicidin, which is encoded by the primary vitamin $\mathrm{D}$ target gene CAMP and rapidly destroys the lipoprotein membranes of microbes (Gombart et al., 2005). Transcriptome-wide analysis indicated that in human monocytes a few hundred additional genes respond to vitamin D (Seuter et al., 2016). This review will present the epigenome- and transcriptome-wide response of the monocytic cell line THP-1 to vitamin D as a paradigm for distinguishing four regulatory scenarios of vitamin $\mathrm{D}$ target genes.

\section{THE TRANSCRIPTION FACTOR VDR}

Larger amounts of VDR protein are found in intestine, kidneys, skin, parathyroid gland and pituitary gland, but also most of the other 400 tissues and cell types of the human body, including those of the innate and adaptive immune system, show some VDR expression ${ }^{1}$. Since transcription factors, such as VDR, do not need high expression levels for their effective function, it can be assumed that most human tissues are sensitive to vitamin D.

Like other members of the nuclear receptor superfamily, VDR carries a structurally conserved ligand-binding domain, the inner surface of which forms a ligand-binding pocket that snugly encloses the molecule $1,25(\mathrm{OH})_{2} \mathrm{D}_{3}$ (Molnár et al., 2006). In turn, VDR interacts via the outer surface of the ligand-binding domain with other nuclear proteins, such as histone acetyltransferases (Herdick and Carlberg, 2000), co-repressors contacting histone deacetylases (Polly et al., 2000), lysine demethylases (Pereira et al., 2011) and chromatin remodelers (Wei et al., 2018). These proteins either form large protein complexes with VDR or their genes are primary or secondary targets of vitamin D.

The special feature of transcription factors, such as VDR, is their ability to bind in a sequence-specific fashion to genomic DNA. VDR preferentially binds as a heterodimeric complex with its partner nuclear receptor retinoid $\mathrm{X}$ receptor $(\mathrm{RXR})$ to a direct repeat of the hexameric motif A/GGG/TTC/GA spaced by three nucleotides, which is referred to as a DR3-type response element (Carlberg et al., 1993; Figure 1). The genome-wide binding pattern of VDR, its so-called cistrome, was determined by the method chromatin immunoprecipitation combined with massive parallel sequencing (ChIP-seq). In human the VDR cistrome had been figured out in lymphocytes (Ramagopalan et al., 2010), colorectal cancer cells (Meyer et al., 2012), hepatic stellate cells (Ding et al., 2013) and macrophage-like cells (Tuoresmäki et al., 2014), but the most comprehensive analysis was done for the monocytic cell line THP-1 (Heikkinen et al., 2011; Neme et al., 2017). After stimulation with VDR ligand in all these in vitro cell culture models some 5,000-20,000 genomic binding sites were observed, which is a 2 - to 10 -fold increase compared to the respective basal condition. Interestingly, the VDR cistrome in THP-1 cells contains a few hundred persistent loci that stay always occupied (Neme et al., 2017). These primary contacts of the human genome with $1,25(\mathrm{OH})_{2} \mathrm{D}_{3}$ are considered as "hotspots" of vitamin D signaling that coordinate the functional consequences of a stimulation with vitamin $\mathrm{D}$ over time. In addition, there are transient VDR binding loci that modulate the response of the epigenome to vitamin $\mathrm{D}$ and support persistent VDR sites. Thus, the genome-wide, ligand-induced binding of VDR to its preferred loci represents the most prominent epigenome-wide effect of vitamin D.

\section{THE CHROMATIN MODEL OF VITAMIN D SIGNALING}

Chromatin is the complex of genomic DNA with nucleosomes that stabilizes the epigenetic landscape of a differentiated cell (Beisel and Paro, 2011). By default chromatin largely restricts the access of transcription factors to promoter and enhancer regions, so that per cell type only some 100-200,000 genomic regions are accessible

\footnotetext{
${ }^{1}$ www.proteinatlas.org/ENSG00000111424-VDR/tissue
} 


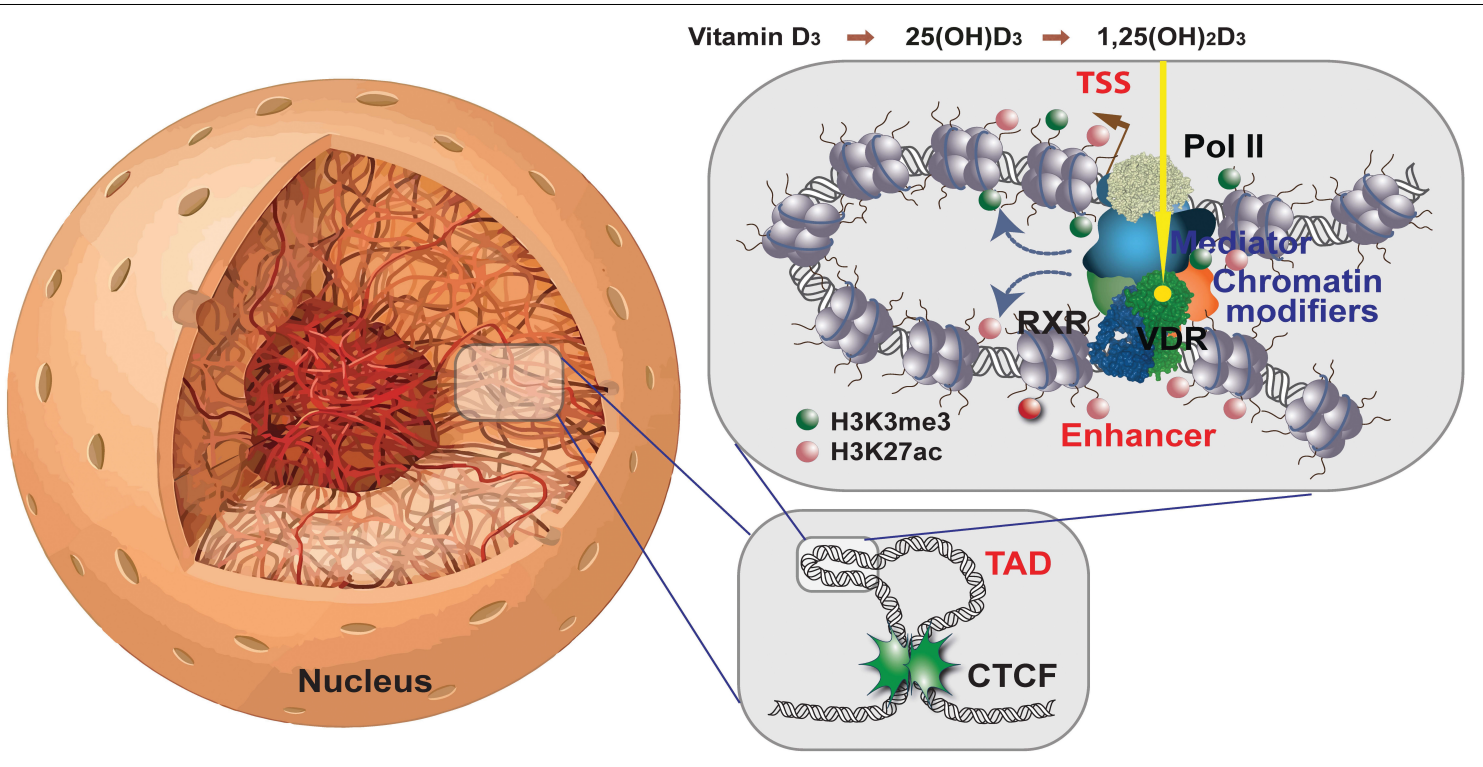

FIGURE 1 | Vitamin D signaling in the context of chromatin. Chromatin within the nucleus forms a 3D architecture (left). Two CTCF proteins bound at adjacent chromatin boundaries form a complex defining a TAD (right bottom). Enhancers and TSS regions that are located within the same TAD can get into physical contact within DNA looping (right top). 1,25(OH) $2 \mathrm{D}_{3}$-activated VDR forms a heterodimeric complex with RXR on enhancer regions carrying appropriate binding sites. In this way, chromatin modifiers are activated that change histone marks (shown here are H3K4me3 modifications marking active TSS regions and $\mathrm{H} 3 \mathrm{~K} 27$ ac indicating active chromatin) and the mediator complex forms a bridge to the basal transcriptional machinery with RNA polymerase II as its core. This finally leads to mRNA transcription of respective vitamin D target genes.

(The Encode-Project-Consortium et al., 2012). The epigenome is represented by (i) more than 100 post-translational modifications, such as acetylations and methylations, of the four nucleosome-forming histone proteins (Carlberg and Molnár, 2016a), (ii) DNA methylation, preferentially within so-called "CpG islands" (Dawson and Kouzarides, 2012) and iii) higher order chromatin structures, such as the three-dimensional organization of the genome into topologically associated domains (TADs) (Carlberg and Molnár, 2016b). Importantly, the epigenome is dynamic, i.e., it can be modulated by environmental signals that influence chromatin modifying enzymes, such as DNA methyltransferases, histone acetyl- and methyltransferases as well as histone deacetylases and demethylases, that write, read or erase marks on genomic DNA and histone proteins.

For the transcription of a gene it is essential that the genomic regions of both its transcription start site (TSS) and the binding sites of the transcription factors controlling the activity of RNA polymerase II, referred to as enhancers, are located within accessible chromatin (Carlberg and Campbell, 2013). Open chromatin can be measured by the method formaldehydeassisted isolation of regulatory elements sequencing (FAIREseq) and some 9,000 sites of accessible chromatin were found to be sensitive to vitamin D stimulation (Seuter et al., 2016). A prominent marker of active chromatin at enhancer regions is the histone modification $\mathrm{H} 3 \mathrm{~K} 27 \mathrm{ac}$, while H3K4me3 labels TSS regions. Interestingly, both types of histone markers are sensitive to vitamin D (Nurminen et al., 2018).

In human, most epigenome-wide data on $1,25(\mathrm{OH})_{2} \mathrm{D}_{3}$ and its receptor VDR have been collected in cells of the innate and adaptive immune system (Carlberg, 2014). Accordingly, the chromatin model of vitamin D signaling, describing the sequential process of vitamin D target gene activation, has been developed based on epigenome- and transcriptome-wide data obtained primarily in THP-1 cells (Carlberg, 2017). As outlined above, the first event in vitamin $\mathrm{D}$ signaling is the genome-wide binding of ligand-activated VDR molecules to enhancer regions that carry suitable binding motifs and are located within accessible chromatin. With the help of pioneer factors, such as PU.1 (Seuter et al., 2017a), CEBPA (Nurminen et al., 2019) and GABPA (Seuter et al., 2018) VDR increases the accessibility of chromatin at and around these enhancer regions. This epigenomic process also involves local changes in the intensity of $\mathrm{H} 3 \mathrm{~K} 27 \mathrm{ac}$ histone markers marking active chromatin at enhancer and TSS regions (Nurminen et al., 2018). Via DNA looping VDR-bound, activate enhancer regions contact TSS regions within the same TAD and trigger the transcription of the respective vitamin $\mathrm{D}$ target genes (Figure 1).

TADs range in size from $30 \mathrm{~kb}$ to $3 \mathrm{Mb}$ and subdivide the human genome into at least 2,000 functionally independent domains (Dixon et al., 2012). Enhancer-TSS contacts are very likely within the same TAD, while interaction with genomic regions outside of a TAD are inhibited by insulating TAD borders that are marked by the transcription factor CTCF (Ali et al., 2016; Figure 1). Thus, VDR-bound enhancers can only modulate the transcription of those genes, which are located within the same TAD. Accordingly, the linear distance between a VDR site within an enhancer and its target TSS is limited by the size of the respective TAD but it can be many hundred $\mathrm{kb}$ (see Supplementary Table $\$ 1$ for examples). 
Interestingly, in THP-1 cells $1,25(\mathrm{OH})_{2} \mathrm{D}_{3}$ stimulation significantly $(p<0.05)$ affects the binding strength of CTCF to TAD anchors making some 600 TADs sensitive of vitamin D (Neme et al., 2016b). Looping of activated DNA-bound VDR to a TSS leads at these promoter regions to an increase in chromatin accessibility as well as of H3K27ac and H3K4me3 marks (Seuter et al., 2016; Nurminen et al., 2019). All these vitamin D-triggered changes in the local chromatin structure at enhancer and promoter regions finally lead to the activation of RNA polymerase II assembled on the respective TSSs and the start of mRNA synthesis. Thus, vitamin D signaling involves a number of epigenome-wide events before there are responses on the level of the transcriptome.

The chromatin model of vitamin D signaling explains the activation of primary vitamin $\mathrm{D}$ target genes, which are the focus of this review. However, there is also a large number of secondary vitamin $\mathrm{D}$ target genes, the activation of which do not directly require $\mathrm{VDR}$ as a central protein. The activity of transcription factors other than VDR and/or chromatin modifying proteins, which are encoded by primary targets of vitamin $\mathrm{D}$, mediate the activation of these secondary vitamin $\mathrm{D}$ target genes. Examples are the transcription factors BCL6, NFE2, POU4F2, and ELF4, which are in THP-1 cells primary vitamin D target genes (Nurminen et al., 2015).

\section{VITAMIN D TARGET GENES IN THP-1 CELLS}

Since more than two decades many research groups have used the human acute monocytic leukemia cell line THP-1 (Tsuchiya et al., 1980) as a model system for investigating the effects of vitamin D-triggered physiological processes in the context of innate immunity. A PubMed search (September 19, 2018) with the keywords "Vitamin D AND THP-1" revealed 161 publications, which were manually inspected whether they report in undifferentiated THP-1 cells statistically significant effects of $1,25(\mathrm{OH})_{2} \mathrm{D}_{3}$ on the mRNA expression of individual genes, as measured by qPCR, or of gene sets, as monitored by microarrays or RNA sequencing (RNA-seq). The attribute "undifferentiated THP-1 cells" was fulfilled by 53 articles, 19 of which were not considered, since they used less quantitative assays, such as Northern blotting, or focused on changes in protein expression by flow cytometry or Western blotting. The remaining 34 publications reported effects of $1,25(\mathrm{OH})_{2} \mathrm{D}_{3}$ on enhancer/TSS regions and/or mRNA expression of in total 107 different genes (Table 1). In reference to a re-analyzed RNA-seq time course (Seuter et al., 2016; Neme et al., 2017; Nurminen et al., 2019), of these genes were classified as primary vitamin $\mathrm{D}$ targets, i.e., their expression changed significantly $(p<0.05)$ within $4 \mathrm{~h}$ after onset of stimulation with $1,25(\mathrm{OH})_{2} \mathrm{D}_{3}$, while 28 genes were secondary vitamin $\mathrm{D}$ targets. However, the transcriptome-wide assay failed to confirm 15 genes, i.e., $14 \%$ of all, as vitamin D target genes. This reflects a well-known discrepancy, which is based mainly on different threshold settings and more strict statistical approaches of the transcriptomewide method.
Next, all publically available transcriptome-wide data for $1,25(\mathrm{OH})_{2} \mathrm{D}_{3}$-stimulated, undifferentiated THP-1 cells were compared. A search of the Gene Expression Omnibus ${ }^{2}$ resulted in the microarray datasets GSE60102 (Heikkinen et al., 2011) and GSE52819 (Verway et al., 2013) as well as in the RNA-seq datasets GSE69284 (Seuter et al., 2016) and GSE119556 (Nurminen et al., 2019). In all four datasets undifferentiated THP-1 cells had been stimulated for $24 \mathrm{~h}$ with $1,25(\mathrm{OH})_{2} \mathrm{D}_{3}$. The comparison of the 3,372 significantly $(p<0.05)$ regulated genes in the microarray from 2011 (Heikkinen et al., 2011) and the 4,532 genes in the microarray from 2013 (Verway et al., 2013) indicated 1,227 common genes (Supplementary Figure S1). 695 of the latter matched with the 3,650 genes reported in the re-analyzed RNAseq dataset from 2016 (Seuter et al., 2016; Neme et al., 2017) and 268 with the 951 genes identified in the RNA-seq dataset from 2018 (Nurminen et al., 2019). Furthermore, both RNA-seq datasets had 273 overlapping genes, 126 of which belonged to the 1,227 common genes found by microarrays.

Taken together, the four transcriptome-wide dataset agreed on 126 genes that were regulated within $24 \mathrm{~h}$ after a stimulation with $1,25(\mathrm{OH})_{2} \mathrm{D}_{3}$. Although all four experimental series had been performed in three biological repeats and only significantly $(p<0.05)$ modulated genes were considered, the rather low number of common genes indicates that the fluctuation in low expressed, moderately regulated genes was very high.

\section{TRANSCRIPTIONAL RESPONSE OF VITAMIN D TARGET GENES}

The recent $1,25(\mathrm{OH})_{2} \mathrm{D}_{3}$-dependent transcriptome analysis (Nurminen et al., 2019) identified in THP-1 cells 951 genes as vitamin D targets, 273 of which overlapped with the reanalyzed earlier RNA-seq dataset (Seuter et al., 2016; Neme et al., 2017; Figure 2A). The latter dataset allowed assigning 189 of the 273 genes $(69 \%)$ as primary vitamin D targets (Supplementary Table S1). Interestingly, 59 (75\% primary) of the 273 common genes had already been characterized by single-gene approaches (Table 1). From the 126 genes that were also found by microarrays about the same proportion (72\%) were primary vitamin D targets (Figure 2A).

The 189 primary vitamin D target genes (Figure 2A) were classified by the fold change (FC) of their mRNA expression after $1,25(\mathrm{OH})_{2} \mathrm{D}_{3}$ stimulation for $24 \mathrm{~h}$ (Supplementary Figure S2). The six genes CD14 (encoding for a toll-like receptor coreceptor), ORM1 (encoding for an acute phase plasma protein), $C A M P, F B P 1$ (encoding for a glucose metabolizing enzyme), CYP26B1 (encoding for an enzyme metabolizing retinoids) and TSPAN18 (encoding for a membrane protein with unclear function) showed a FC larger than 40 and form group A. The FC of further 13 genes composing group $\mathrm{B}$ was in the range of 10 to 40 , while the large group $\mathrm{C}$ comprised 170 genes displaying a FC below 10. Only 5 of the 189 primary vitamin $\mathrm{D}$ target genes are down-regulated, while nearly half of all secondary vitamin D target genes are down-regulated (Neme et al., 2016a). Thus, in

\footnotetext{
${ }^{2}$ www.ncbi.nlm.nih.gov/geo
} 
TABLE 1 | Vitamin D target genes.

\begin{tabular}{|c|c|c|c|}
\hline Gene & Primary? & Type(s) of assays & Citation \\
\hline CAMP & Yes & qPCR & Liu et al., 2007 \\
\hline CYP24A1 & - & qPCR & Liu et al., 2007 \\
\hline CYP24A1 & - & qPCR & Wu et al., 2007 \\
\hline CYP27B1 & - & qPCR & Wu et al., 2007 \\
\hline CD14 & Yes & qPCR & Moeenrezakhanlou et al., 2008 \\
\hline ITGAM & Yes & qPCR & Moeenrezakhanlou et al., 2008 \\
\hline ALOX5 & No & qPCR & Matsunawa et al., 2009 \\
\hline CAMP & Yes & qPCR & Matsunawa et al., 2009 \\
\hline CD14 & Yes & qPCR & Matsunawa et al., 2009 \\
\hline CYP24A1 & - & qPCR & Matsunawa et al., 2009 \\
\hline FANCE & Yes & ChIP-seq \& FAIRE-seq display, qPCR & Seuter et al., 2013b \\
\hline HBEGF & Yes & ChIP-seq \& FAIRE-seq display, qPCR & Seuter et al., 2013b \\
\hline$N F K B I A$ & Yes & ChIP-seq \& FAIRE-seq display, qPCR & Seuter et al., 2013b \\
\hline PDCD1LG2 & Yes & ChIP-seq \& FAIRE-seq display, qPCR & Seuter et al., 2013b \\
\hline TMEM37 & Yes & ChIP-seq \& FAIRE-seq display, qPCR & Seuter et al., 2013b \\
\hline BHLHE4O & - & ChIP-seq \& FAIRE-seq display, qPCR & Seuter et al., 2013c \\
\hline CAMP & Yes & ChIP-seq \& FAIRE-seq display, qPCR & Seuter et al., 2013a \\
\hline CD93 & Yes & ChIP-seq \& FAIRE-seq display, qPCR & Seuter et al., 2013a \\
\hline DUSP10 & Yes & ChIP-seq \& FAIRE-seq display, qPCR & Seuter et al., 2013a \\
\hline HBEGF & Yes & ChIP-seq \& FAIRE-seq display, qPCR & Seuter et al., 2013a \\
\hline NFKBIA & Yes & ChIP-seq \& FAIRE-seq display, qPCR & Seuter et al., 2013a \\
\hline$T H B D$ & Yes & ChIP-seq \& FAIRE-seq display, qPCR & Seuter et al., 2013a \\
\hline $\operatorname{IL1B}$ & No & qPCR, microarray & Verway et al., 2013 \\
\hline ASAP2 & Yes & ChIP-seq display, qPCR & Seuter et al., 2014b \\
\hline$Y W H A Q$ & No & ChIP-seq display, qPCR & Seuter et al., 2014b \\
\hline SEPT3 & Yes & ChIP-seq \& FAIRE-seq display, qPCR & Seuter et al., 2014a \\
\hline SFT2D1 & Yes & ChIP-seq \& FAIRE-seq display, qPCR & Seuter et al., 2014a \\
\hline SP100 & Yes & ChIP-seq \& FAIRE-seq display, qPCR & Seuter et al., 2014a \\
\hline ZFP36 & Yes & ChIP-seq \& FAIRE-seq display, qPCR & Seuter et al., 2014a \\
\hline TNF & - & qPCR & Reeves et al., 2014 \\
\hline ASAP2 & Yes & ChIP-seq display & Tuoresmäki et al., 2014 \\
\hline CAMP & Yes & ChIP-seq display & Tuoresmäki et al., 2014 \\
\hline DENND6B & Yes & ChIP-seq display & Tuoresmäki et al., 2014 \\
\hline NINJ1 & Yes & ChIP-seq display & Tuoresmäki et al., 2014 \\
\hline PTGER3 & - & ChIP-seq display & Tuoresmäki et al., 2014 \\
\hline SP100 & Yes & ChIP-seq display & Tuoresmäki et al., 2014 \\
\hline TBP & No & ChIP-seq display & Tuoresmäki et al., 2014 \\
\hline TRAK1 & Yes & ChIP-seq display & Tuoresmäki et al., 2014 \\
\hline TLR10 & - & qPCR & Verma et al., 2014 \\
\hline CD97 & Yes & ChIP-seq \& FAIRE-seq display, qPCR & Wilfinger et al., 2014 \\
\hline LRRC8A & Yes & ChIP-seq \& FAIRE-seq display, qPCR & Wilfinger et al., 2014 \\
\hline NRIP1 & Yes & ChIP-seq \& FAIRE-seq display, qPCR & Wilfinger et al., 2014 \\
\hline SLC37A2 & Yes & ChIP-seq \& FAIRE-seq display, qPCR & Wilfinger et al., 2014 \\
\hline TREM1 & Yes & qPCR & Hosoda et al., 2015 \\
\hline$B C L 6$ & Yes & Microarray, qPCR & Nurminen et al., 2015 \\
\hline ELF4 & Yes & ChIP-seq \& FAIRE-seq display, microarray, qPCR & Nurminen et al., 2015 \\
\hline FUCA1 & Yes & ChIP-seq \& FAIRE-seq display, microarray, qPCR & Nurminen et al., 2015 \\
\hline ITGAM & Yes & ChIP-seq \& FAIRE-seq display, microarray, qPCR & Nurminen et al., 2015 \\
\hline LPGAT1 & No & ChIP-seq \& FAIRE-seq display, microarray, qPCR & Nurminen et al., 2015 \\
\hline$L P P$ & - & Microarray, qPCR & Nurminen et al., 2015 \\
\hline$L R R C 25$ & Yes & ChIP-seq \& FAIRE-seq display, microarray, qPCR & Nurminen et al., 2015 \\
\hline NFE2 & Yes & ChIP-seq \& FAIRE-seq display, microarray, qPCR & Nurminen et al., 2015 \\
\hline POU4F2 & Yes & ChIP-seq \& FAIRE-seq display, microarray, qPCR & Nurminen et al., 2015 \\
\hline
\end{tabular}


TABLE 1 | Continued

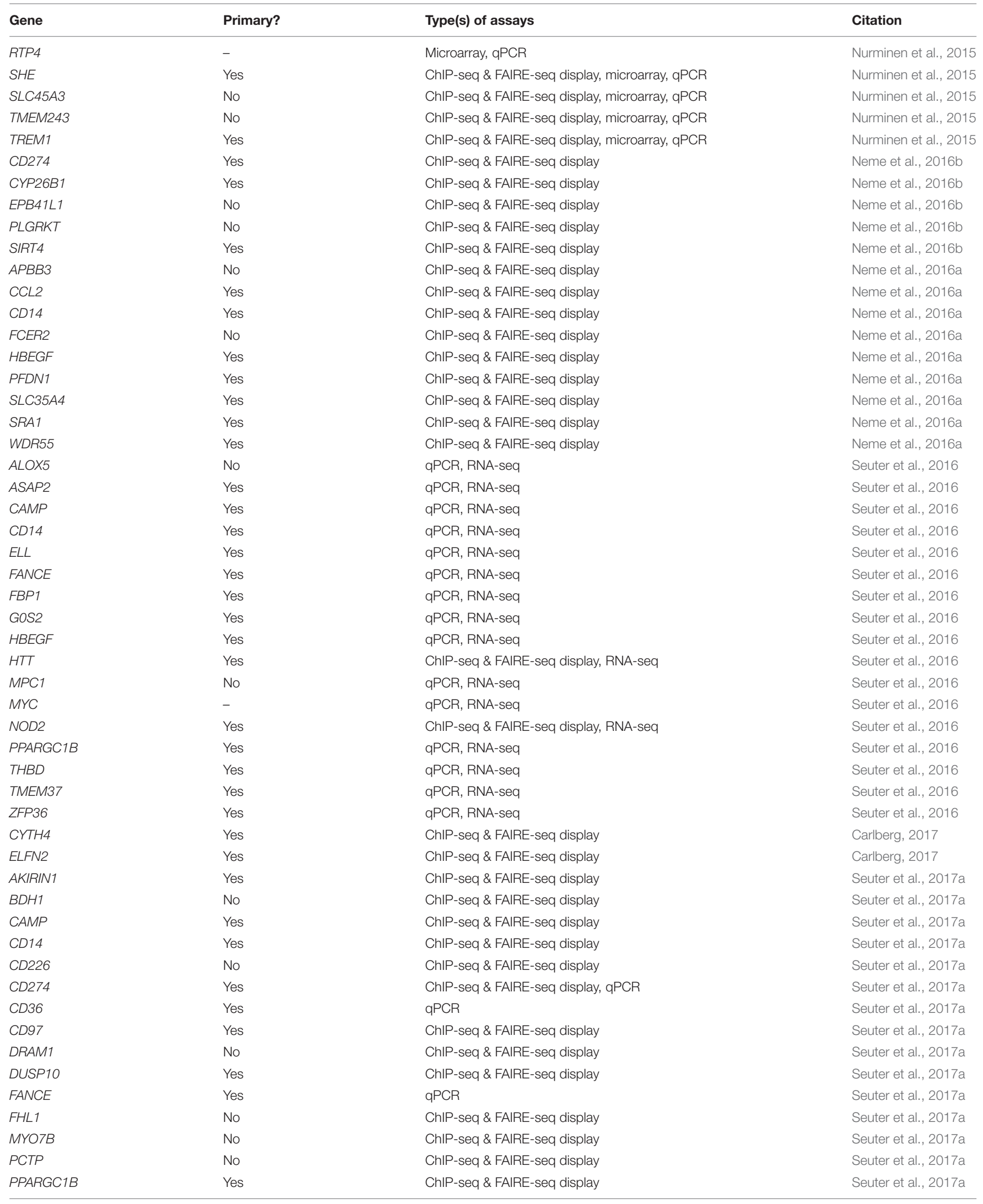


TABLE 1 | Continued

\begin{tabular}{|c|c|c|c|}
\hline Gene & Primary? & Type(s) of assays & Citation \\
\hline$R T C B$ & - & ChIP-seq \& FAIRE-seq display & Seuter et al., 2017a \\
\hline SLC37A2 & Yes & ChIP-seq \& FAIRE-seq display & Seuter et al., 2017a \\
\hline SPI1 & - & qPCR & Seuter et al., 2017a \\
\hline AGPAT1 & Yes & ChIP-seq \& FAIRE-seq display & Carlberg, 2018 \\
\hline CD14 & Yes & ChIP-seq \& FAIRE-seq display & Nurminen et al., 2018 \\
\hline CLMN & Yes & ChIP-seq \& FAIRE-seq display & Nurminen et al., 2018 \\
\hline TMEM37 & Yes & ChIP-seq \& FAIRE-seq display & Nurminen et al., 2018 \\
\hline CD14 & Yes & ChIP-seq \& FAIRE-seq display & Seuter et al., 2018 \\
\hline COQ3 & No & ChIP-seq \& FAIRE-seq display & Seuter et al., 2018 \\
\hline DND1 & Yes & ChIP-seq \& FAIRE-seq display & Seuter et al., 2018 \\
\hline NDUFA2 & No & ChIP-seq \& FAIRE-seq display & Seuter et al., 2018 \\
\hline PSMB1 & No & ChIP-seq \& FAIRE-seq display & Seuter et al., 2018 \\
\hline SLC25A15 & No & ChIP-seq \& FAIRE-seq display & Seuter et al., 2018 \\
\hline SLC52A2 & No & ChIP-seq \& FAIRE-seq display & Seuter et al., 2018 \\
\hline TBP & No & ChIP-seq \& FAIRE-seq display & Seuter et al., 2018 \\
\hline TMCO6 & Yes & ChIP-seq \& FAIRE-seq display & Seuter et al., 2018 \\
\hline WDR55 & Yes & ChIP-seq \& FAIRE-seq display & Seuter et al., 2018 \\
\hline ZNF44 & Yes & ChIP-seq \& FAIRE-seq display & Seuter et al., 2018 \\
\hline ACSL1 & Yes & ChIP-seq \& FAIRE-seq display, RNA-seq & Nurminen et al., 2019 \\
\hline CD14 & Yes & ChIP-seq \& FAIRE-seq display, RNA-seq & Nurminen et al., 2019 \\
\hline$C D A$ & No & ChIP-seq \& FAIRE-seq display, RNA-seq & Nurminen et al., 2019 \\
\hline FBP1 & Yes & ChIP-seq \& FAIRE-seq display, RNA-seq & Nurminen et al., 2019 \\
\hline GOS2 & Yes & ChIP-seq \& FAIRE-seq display, RNA-seq & Nurminen et al., 2019 \\
\hline GLIPR1 & Yes & ChIP-seq \& FAIRE-seq display, RNA-seq & Nurminen et al., 2019 \\
\hline INSR & Yes & ChIP-seq \& FAIRE-seq display, RNA-seq & Nurminen et al., 2019 \\
\hline ITSN1 & Yes & ChIP-seq \& FAIRE-seq display, RNA-seq & Nurminen et al., 2019 \\
\hline$K L H D C 8 B$ & No & ChIP-seq \& FAIRE-seq display, RNA-seq & Nurminen et al., 2019 \\
\hline NFKBIA & Yes & ChIP-seq \& FAIRE-seq display, RNA-seq & Nurminen et al., 2019 \\
\hline PNPLA1 & No & ChIP-seq \& FAIRE-seq display, RNA-seq & Nurminen et al., 2019 \\
\hline SERINC2 & Yes & ChIP-seq \& FAIRE-seq display, RNA-seq & Nurminen et al., 2019 \\
\hline SOAT1 & No & ChIP-seq \& FAIRE-seq display, RNA-seq & Nurminen et al., 2019 \\
\hline SSH1 & Yes & ChIP-seq \& FAIRE-seq display, RNA-seq & Nurminen et al., 2019 \\
\hline
\end{tabular}

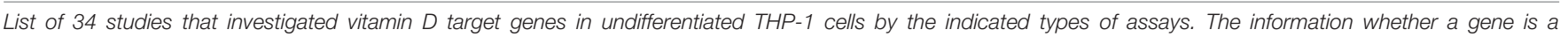

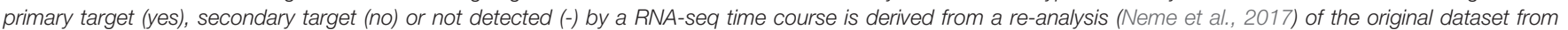
Seuter et al. (2016).

contrast to up-regulation, the mechanism of down-regulation of vitamin $\mathrm{D}$ target genes is mostly an indirect, multi-step process.

\section{FUNCTIONAL PROFILE OF VITAMIN D TARGET GENES}

The biologically most important question of the transcriptomewide analysis was, how the action of the complete set of vitamin $\mathrm{D}$ target genes translates into physiological functions of human monocytes. Therefore, gene ontology analysis was applied, in order to identify statistically significant overrepresentation of vitamin D target genes in biological pathways. Using the webtool Enrichr (Chen et al., 2013) for the gene lists with 951, 273, and 126 members (Figure 2A) provided the result that the top five biological pathways, such as "neutrophil activation," "positive regulation of TNF production," "inflammatory response," "neutrophil degranulation," "negative regulation of $\mathrm{T}$ cell proliferation" and "positive regulation of cytokine secretion," related for each of the three gene sets to key functions in innate immunity (Figure 2B). This result could have been expected, since monocytes represent the major cell type of the innate immune system. Nevertheless, it is important to confirm that the key immune-related functions of monocytes are supported by vitamin D.

In summary, independent of the size of the tested target gene set, gene ontology analysis indicates that the modulation of innate immunity is the main physiological outcome of a vitamin $\mathrm{D}$ stimulation of human monocytes.

\section{CLASSIFICATION OF PRIMARY VITAMIN D TARGET GENES}

Each of the 189 primary vitamin D target genes (Supplementary Table S1) was manually inspected for the epigenomic profile at 


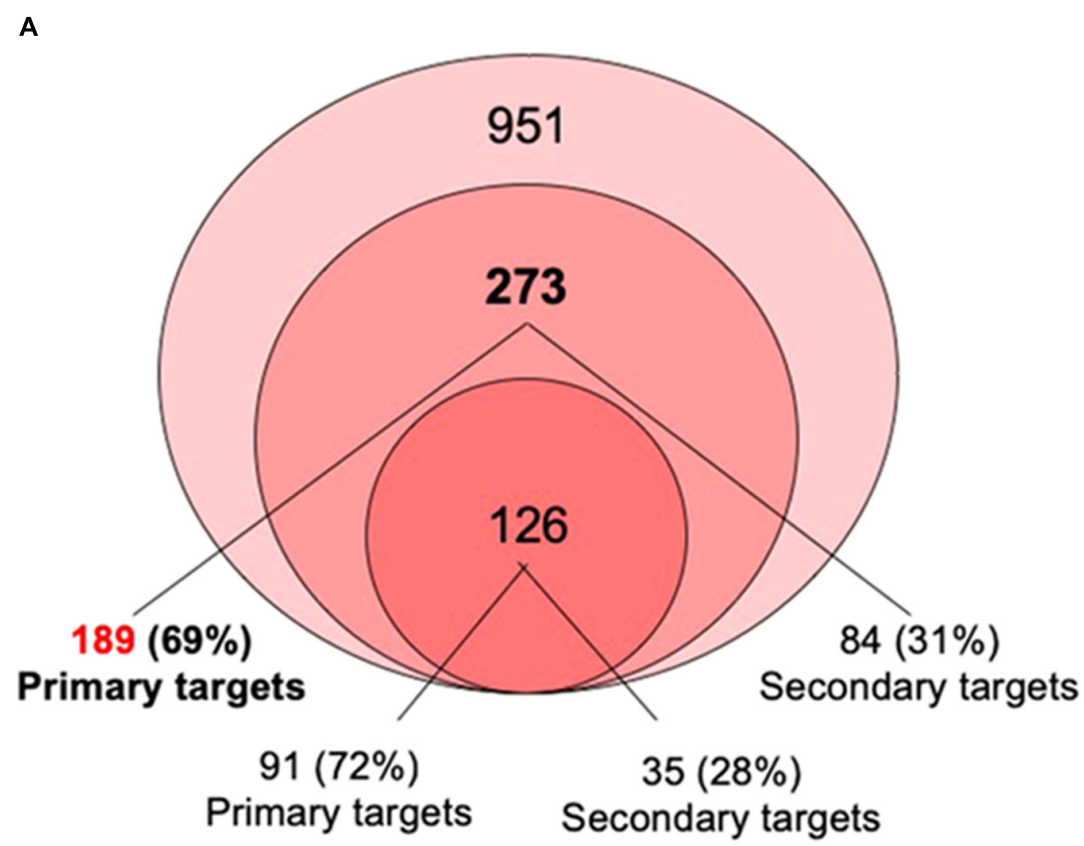

B Gene ontology analysis

1. Neutrophil activation involved in immune response

2. Neutrophil mediated immunity

3. Inflammatory response

4. Neutrophil degranulation

5. Positive regulation of cytokine secretion

1. Neutrophil activation involved in immune response

2. Negative regulation of activated $\mathrm{T}$ cell proliferation

3. Neutrophil degranulation

4. Positive regulation of intracellular signal transduction

5. Neutrophil mediated immunity

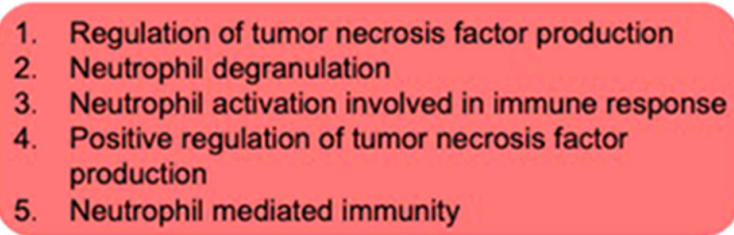

FIGURE 2 | Gene ontology analysis. The most recent 1,25(OH) ${ }_{2} \mathrm{D}_{3}$-dependent transcriptome dataset of THP-1 cells (Nurminen et al., 2019 ) identified 951 genes, 273 of which overlap with the re-analyzed first RNA-seq dataset (Seuter et al., 2016; Neme et al., 2017) (A). 69\% of the 273 genes are primary vitamin D targets (189 genes listed in Supplementary Table S1), while from the 126 genes that were also found by microarrays $72 \%$ are primary vitamin D targets. Gene ontology analysis using the webtool Enrichr (Chen et al., 2013) was performed for the lists of 951, 273, and 126 members and indicated that the top five biological pathways for each of the three gene sets relate to innate immunity (B).

its TSS and enhancer regions, such as (i) occurrence of H3K4me3 and H3K27ac marks, (ii) binding of VDR, PU.1 and CEBPA as well as (iii) the significant $(p<0.05)$ modulation of both type of datasets by $1,25(\mathrm{OH})_{2} \mathrm{D}_{3}$. The whole TAD region of each primary target gene was screened for the most prominent enhancer. For 17 genes the enhancer was found in a distance of below $1 \mathrm{~kb}$ from the TSS, while in contrast for 23 genes it located more than $100 \mathrm{~kb}$ apart (Supplementary Table S1). Importantly, 160 of the 189 genes showed H3K4me3 marks at their TSS regions and could be segregated into four classes (Supplementary Table S1 and Figure 3), which were defined as follows: VDR bound to the enhancer regions of each of these 160 genes, but only 


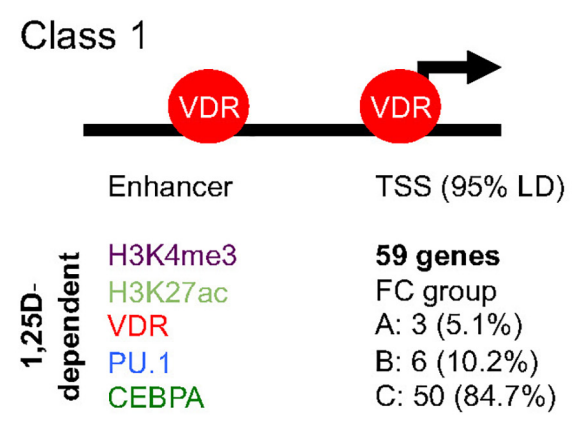

Avg. FC: 11.24

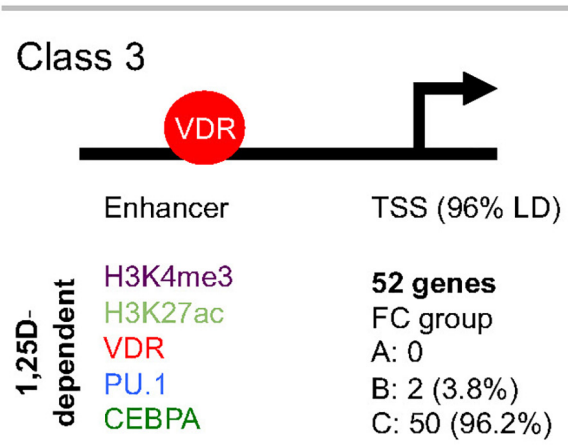

Avg. FC: 3.67

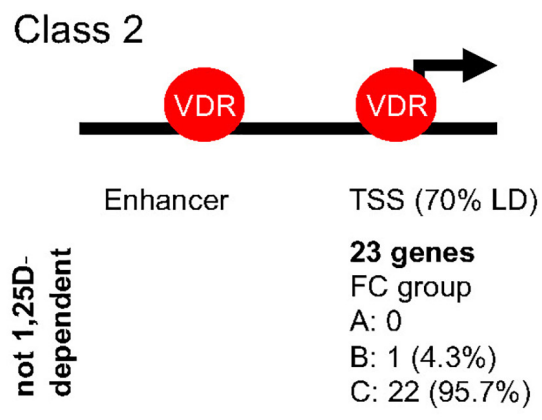

Avg. FC: 3.53
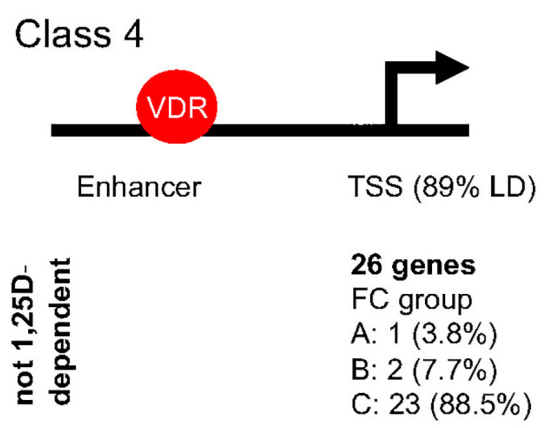

Avg. FC: 6.69

FIGURE 3 | Classification of primary vitamin D target genes. The 160 primary vitamin D target genes that show H3K4me3 marks at their TSS regions can be segregated into four classes. The structure of the genes into TSS and enhancer regions is schematically depicted. Genes of classes 1 and 2 display VDR binding to their TSS regions, while for those of classes 3 and 4 no VDR binding can be detected. The 59 genes of class 1 and the 52 genes of class 3 have significant $(p<0.05)$ effects of $1,25(\mathrm{OH})_{2} \mathrm{D}_{3}$ on $\mathrm{H} 3 \mathrm{~K} 4 \mathrm{me}$, H3K27ac, VDR, PU.1 and/or CEBPA binding strength on their enhancer region, while this is not observed for the 23 genes of class 2 and the 26 genes of class 4 . In addition, for the respective classes the distribution of the genes of into the fold change (FC) groups A, B and $\mathrm{C}$ (Supplementary Figure S2) as well as the average FC is indicated.

the 82 genes of classes 1 and 2 displayed VDR binding also to their TSS regions, while for the 78 genes of classes 3 and 4 no VDR was detected at their promoters. In addition to genes with enhancer regions close to their promoters $(82 \%$ of which are class 1 genes), VDR could be detected at TSS regions, because it is assumed to loop from an enhancer region rather than due to direct promoter binding. Thus, probably on class 3 and 4 genes no VDR was detected, because the protein did not make long and frequent enough contacts, in order to create at the respective TSS regions a significant mark in the VDR cistrome. However, $1,25(\mathrm{OH})_{2} \mathrm{D}_{3}$-triggered increases of $\mathrm{H} 3 \mathrm{~K} 4 \mathrm{me} 3$ marks at TSS regions of 56 class 3 and 4 genes provided at least indirect evidence for a looping of enhancer-bound VDR to these promoter regions.

The 59 genes of class 1 were distinguished from the 23 genes of class 2 by experiencing significant $(p<0.05)$ effects of ligand on the intensity of H3K4me3 and H3K27ac marks or the binding strength of VDR, PU.1 and/or CEBPA at their enhancer regions (Figure 3). By the same kind of $1,25(\mathrm{OH})_{2} \mathrm{D}_{3}$-sensitivity at their enhancer regions the 52 genes of class 3 differed from the 26 genes of class 4 . Representative examples for the differential epigenomic profiles at enhancer and TSS regions are the regulatory scenarios of the class 1 gene TMEM37 (encoding for a stabilizer of calcium channels), the class 2 gene LILRB4 (encoding for a membrane receptor), the class 3 gene TFE3 (encoding for a transcription factor) and the class 4 gene CYP26B1 (Supplementary Figure S3). In contrast, for 29 genes no specific epigenomic pattern on their TSS and enhancer regions had been detected, i.e., they remain unclassified for their epigenomic profile (Supplementary Table S1).

The 59 genes of class 1 showed with 11.24 the highest average FC, because three of them belong to the highly upregulated group A and six to the strongly up-regulated group B (Figure 3). Second ranking in average FC (6.69) were the 26 genes of class 4, since they comprise one group A gene and two group B genes. In contrast, the 52 class 3 genes averaged only in a FC of 3.67 (two group B genes and 50 moderately regulated group $\mathrm{C}$ genes) and the 23 class 2 gene even only in an average FC of 3.53 (one group B gene and 22 group $\mathrm{C}$ genes).

Taken together, 160 of the 189 primary vitamin $\mathrm{D}$ target genes could be segregated into four classes by the epigenomic profile of their enhancer and TSS regions. The key distinguishing protein of the respective gene regulatory scenarios was VDR bound to TSS and enhancer regions and being supported at enhancers by the pioneer transcription factors PU.1 and CEBPA. 


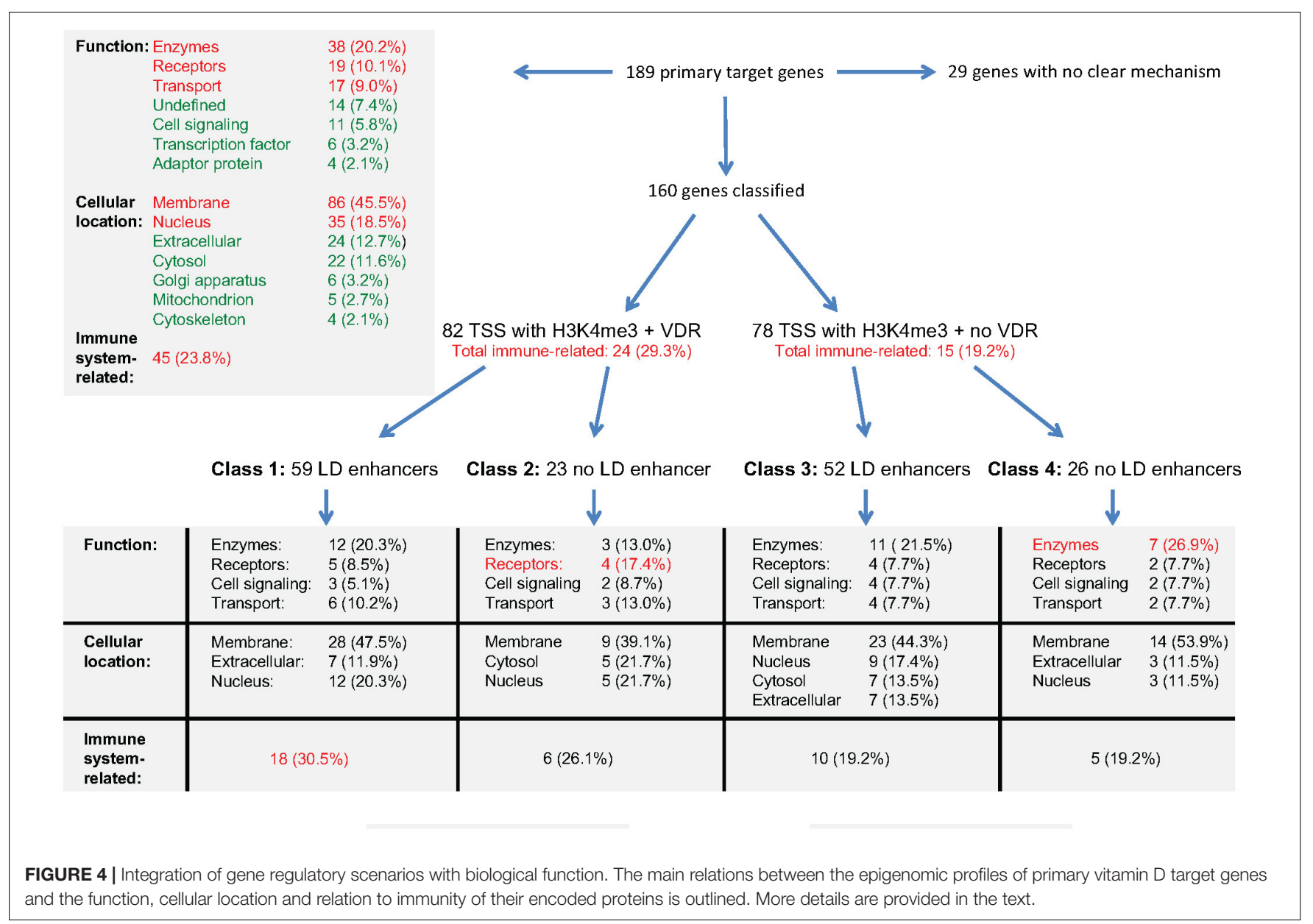

\section{RELATION OF GENE REGULATORY SCENARIOS TO BIOLOGICAL FUNCTION}

The integration of the transcriptome- and epigenome-wide datasets available for vitamin D-triggered human monocytes can be summarized as outlined in Figure 4. On the transcriptomelevel, a stimulation of THP- 1 cells with $1,25(\mathrm{OH})_{2} \mathrm{D}_{3}$ has significant $(p<0.05)$ direct effects on the expression of 189 genes, 184 of which are up-regulated (Supplementary Table S1). The proteins encoded by these genes mainly act as enzymes (20\%), receptors (10\%) and transporters (9\%) and their most prominent locations are membranes (46\%) and the nucleus (19\%). Nearly a fourth $(23.8 \%)$ of these primary vitamin D target genes are directly involved in the function of the immune system. On the epigenome level, 160 of the 189 genes can be classified into four gene regulatory scenarios, while 29 genes (15\%) remain unclassified. The 82 genes with VDR binding at their TSS region show with $29.3 \%$ a higher rate of immune system related function than the 78 genes without detectable VDR (19.2\%). Accordingly, the main characteristic of the 59 class 1 genes is the relation of their function to the immune system. In contrast, in absence of significant effects of $1,25(\mathrm{OH})_{2} \mathrm{D}_{3}$ on enhancer regions, as it applies for classes 2 and 4, preferentially genes with metabolic functions are found.
In summary, immune system-related genes of class 1 are supported by a gene regulatory scenario that allows a prominent up-regulation by vitamin $\mathrm{D}$, while genes related to cellular metabolism show less vitamin D sensitive epigenomic profiles.

\section{FUTURE PERSPECTIVE: TRANSLATION TO PRIMARY CELLS}

In the vitamin D intervention study VitDbol (NCT02063334, ClinicalTrials.gov) healthy human adults were treated once with high dose of vitamin $\mathrm{D}_{3}(2,000 \mu \mathrm{g})$ and blood samples were taken before and $24 \mathrm{~h}$ later (Vukic et al., 2015). Peripheral blood mononuclear cells (PBMCs, a mixture of the vitamin $\mathrm{D}$-responsive cell types monocytes, $\mathrm{T}$ and $\mathrm{B}$ lymphocytes) were isolated in less than an hour after drawing blood, i.e., the epigenome and transcriptome status of the cells was as close as possible to the in vivo situation (Carlberg, 2016). Excluding any further in vitro culture, RNA and chromatin were prepared, in order to test changes in gene expression by qPCR (Vukic et al., 2015) and RNAseq (Neme et al., 2019) as well as alterations in chromatin accessibility at selected genomic regions (Seuter et al., 2017b) and 
genome-wide (Carlberg et al., 2018). Based on five tested individuals the expression of 702 genes changed significantly $(p<0.05)$ in response to vitamin $\mathrm{D}_{3}$ (Neme et al., 2019), $26 \%$ of which were also observed in THP-1 cells as vitamin D target genes (Neme et al., 2017). Although the latter genes were primarily secondary targets, it indicates that the response of PBMCs in vivo to a vitamin $\mathrm{D}_{3}$ bolus resembles that of THP1 cells in vitro to a $1,25(\mathrm{OH})_{2} \mathrm{D}_{3}$ bolus. Similarly, the vitamin $\mathrm{D}_{3}$ bolus significantly $(p<0.05)$ changes in vivo the chromatin accessibility at 853 genomic regions (Carlberg et al., 2018), 87\% of which were also $1,25(\mathrm{OH})_{2} \mathrm{D}_{3}$ responsive in THP-1 cells (Seuter et al., 2016).

Taken together, there is reasonable overlap in the transcriptome- and epigenome-wide vitamin D response of PBMCs in vivo and THP-1 cells in vitro, i.e., principles of vitamin D signaling described in this review for THP-1 cells may also be extrapolated to primary cells, such as PBMCs. Primary monocytes would be most appropriate cellular target, for which, however, no vitamin D-triggered epigenome-wide data exists.

\section{CONCLUSION}

To date, most epigenome- and transcriptome-wide data are available for the actions of vitamin $\mathrm{D}$ in cells of the hematopoietic system (Carlberg, 2014). The integration of these

\section{REFERENCES}

Ali, T., Renkawitz, R., and Bartkuhn, M. (2016). Insulators and domains of gene expression. Curr. Opin. Genet. Dev. 37, 17-26. doi: 10.1016/j.gde.2015. 11.009

Beisel, C., and Paro, R. (2011). Silencing chromatin: comparing modes and mechanisms. Nat. Rev. Genet. 12, 123-135. doi: 10.1038/nrg2932

Bendik, I., Friedel, A., Roos, F. F., Weber, P., and Eggersdorfer, M. (2014). Vitamin D: a critical and essential micronutrient for human health. Front. Physiol. 5:248. doi: 10.3389/fphys.2014.00248

Bouillon, R., and Suda, T. (2014). Vitamin D: calcium and bone homeostasis during evolution. Bonekey Rep. 3:480. doi: 10.1038/bonekey.2013.214

Carlberg, C. (2014). Genome-wide (over)view on the actions of vitamin D. Front. Physiol. 5:167. doi: 10.3389/fphys.2014.00167

Carlberg, C. (2016). Molecular approaches for optimizing vitamin D supplementation. Vitam. Horm. 100, 255-271. doi: 10.1016/bs.vh.2015.10.001

Carlberg, C. (2017). Molecular endocrinology of vitamin D on the epigenome level. Mol. Cell. Endocrinol. 453, 14-21. doi: 10.1016/j.mce.2017.03.016

Carlberg, C. (2018). Vitamin D genomics: from in vitro to in vivo. Front. Endocrinol. 9:250. doi: 10.3389/fendo.2018.00250

Carlberg, C., Bendik, I., Wyss, A., Meier, E., Sturzenbecker, L. J., Grippo, J. F., et al. (1993). Two nuclear signalling pathways for vitamin D. Nature 361, 657-660. doi: $10.1038 / 361657 \mathrm{a} 0$

Carlberg, C., and Campbell, M. J. (2013). Vitamin D receptor signaling mechanisms: integrated actions of a well-defined transcription factor. Steroids 78, 127-136. doi: 10.1016/j.steroids.2012.10.019

Carlberg, C., and Molnár, F. (2016a). The Epigenome. Mechanisms of Gene Regulation, 2nd Edn. Berlin: Springer, 159-172.

Carlberg, C., and Molnár, F. (2016b). The Impact of Chromatin. Mechanisms of Gene Regulation, 2nd Edn. Berlin: Springer, 17-34.

Carlberg, C., Seuter, S., Nurmi, T., Tuomainen, T. P., Virtanen, J. K., and Neme, A. (2018). In vivo response of the human epigenome to vitamin D: a proof-ofprinciple study. J. Steroid. Biochem. Mol. Biol. 180, 142-148. doi: 10.1016/j. jsbmb.2018.01.002 large datasets led to the chromatin model of vitamin D signaling (Carlberg, 2017), which was extended in this summary of recent data by the introduction of four different classes of primary vitamin $\mathrm{D}$ target genes. The respective gene regulatory scenarios distinguish prominent vitamin $\mathrm{D}$ response of genes related to immune function and moderate response of genes involved in cellular metabolism.

\section{AUTHOR CONTRIBUTIONS}

VN and CC designed the project. VN, SS, and CC analyzed the data. CC wrote the manuscript.

\section{FUNDING}

This work was supported by the Academy of Finland (\#267067) to CC and a stipend of the Doctoral School in Molecular Medicine of the University of Eastern Finland to VN.

\section{SUPPLEMENTARY MATERIAL}

The Supplementary Material for this article can be found online at: https://www.frontiersin.org/articles/10.3389/fphys. 2019.00194/full\#supplementary-material

Chen, E. Y., Tan, C. M., Kou, Y., Duan, Q., Wang, Z., Meirelles, G. V., et al. (2013). Enrichr: interactive and collaborative HTML5 gene list enrichment analysis tool. BMC Bioinformatics 14:128. doi: 10.1186/1471-2105-14-128

Dawson, M. A., and Kouzarides, T. (2012). Cancer epigenetics: from mechanism to therapy. Cell 150, 12-27. doi: 10.1016/j.cell.2012.06.013

Ding, N., Yu, R. T., Subramaniam, N., Sherman, M. H., Wilson, C., Rao, R., et al. (2013). A vitamin D receptor/SMAD genomic circuit gates hepatic fibrotic response. Cell 153, 601-613. doi: 10.1016/j.cell.2013.03.028

Dixon, J. R., Selvaraj, S., Yue, F., Kim, A., Li, Y., Shen, Y., et al. (2012). Topological domains in mammalian genomes identified by analysis of chromatin interactions. Nature 485, 376-380. doi: 10.1038/nature11082

Gombart, A. F., Borregaard, N., and Koeffler, H. P. (2005). Human cathelicidin antimicrobial peptide (CAMP) gene is a direct target of the vitamin D receptor and is strongly up-regulated in myeloid cells by 1,25-dihydroxyvitamin D3. FASEB J. 19, 1067-1077. doi: 10.1096/fj.04-3284com

Grad, R. (2004). Cod and the consumptive: a brief history of cod-liver oil in the treatment of pulmonary tuberculosis. Pharm. Hist. 46, 106-120.

Hart, P. H., Gorman, S., and Finlay-Jones, J. J. (2011). Modulation of the immune system by UV radiation: more than just the effects of vitamin D? Nat. Rev. Immunol. 11, 584-596. doi: 10.1038/nri3045

Haussler, M. R., Whitfield, G. K., Kaneko, I., Haussler, C. A., Hsieh, D., Hsieh, J.-C., et al. (2013). Molecular mechanisms of vitamin D action. Calcif. Tissue Int. 92, 77-98. doi: 10.1007/s00223-012-9619-0

Heikkinen, S., Väisänen, S., Pehkonen, P., Seuter, S., Benes, V., and Carlberg, C. (2011). Nuclear hormone $1 \alpha, 25$-dihydroxyvitamin D3 elicits a genome-wide shift in the locations of VDR chromatin occupancy. Nucleic Acids Res. 39, 9181-9193. doi: 10.1093/nar/gkr654

Herdick, M., and Carlberg, C. (2000). Agonist-triggered modulation of the activated and silent state of the vitamin D3 receptor by interaction with corepressors and co-activators. J. Mol. Biol. 304, 793-801. doi: 10.1006/jmbi.2000. 4267

Hewison, M. (2012). An update on vitamin D and human immunity. Clin. Endocrinol. 76, 315-325. doi: 10.1111/j.1365-2265.2011. 04261.x 
Holick, M. F. (1981). The cutaneous photosynthesis of previtamin D3: a unique photoendocrine system. J. Invest Ermatol. 77, 51-58. doi: 10.1111/1523-1747. ep12479237

Holick, M. F. (2011). Photobiology of Vitamin D. Boston, MA: Boston University, 13-22. doi: 10.1016/B978-0-12-381978-9.10002-2

Hosoda, H., Tamura, H., and Nagaoka, I. (2015). Evaluation of the lipopolysaccharide-induced transcription of the human TREM-1 gene in vitamin D3-matured THP-1 macrophage-like cells. Int. J. Mol. Med. 36, 1300-1310. doi: 10.3892/ijmm.2015.2349

Liu, P. T., Stenger, S., Li, H., Wenzel, L., Tan, B. H., Krutzik, S. R., et al. (2006). Tolllike receptor triggering of a vitamin $\mathrm{D}$-mediated human antimicrobial response. Science 311, 1770-1773. doi: 10.1126/science. 1123933

Liu, P. T., Stenger, S., Tang, D. H., and Modlin, R. L. (2007). Cutting edge: vitamin D-mediated human antimicrobial activity against Mycobacterium tuberculosis Is dependent on the induction of cathelicidin. J. Immunol. 179, 2060-2063. doi: 10.4049/jimmunol.179.4.2060

Matsunawa, M., Amano, Y., Endo, K., Uno, S., Sakaki, T., Yamada, S., et al. (2009). The aryl hydrocarbon receptor activator benzo[a]pyrene enhances vitamin D3 catabolism in macrophages. Toxicol. Sci. 109, 50-58. doi: 10.1093/toxsci/ kfp044

Meyer, M. B., Goetsch, P. D., and Pike, J. W. (2012). VDR/RXR and TCF4/betacatenin cistromes in colonic cells of colorectal tumor origin: impact on c-FOS and c-MYC gene expression. Mol. Endocrinol. 26, 37-51. doi: 10.1210/me.20111109

Moeenrezakhanlou, A., Shephard, L., Lam, L., and Reiner, N. E. (2008). Myeloid cell differentiation in response to calcitriol for expression CD11b and CD14 is regulated by myeloid zinc finger-1 protein downstream of phosphatidylinositol 3-kinase. J. Leukoc. Biol. 84, 519-528. doi: 10.1189/jlb.1207833

Molnár, F., Peräkylä, M., and Carlberg, C. (2006). Vitamin D receptor agonists specifically modulate the volume of the ligand-binding pocket. J. Biol. Chem. 281, 10516-10526. doi: 10.1074/jbc.M513609200

Neme, A., Nurminen, V., Seuter, S., and Carlberg, C. (2016a). The vitamin D-dependent transcriptome of human monocytes. J. Steroid Biochem. Mol. Biol. 164, 180-187. doi: 10.1016/j.jsbmb.2015.10.018

Neme, A., Seuter, S., and Carlberg, C. (2016b). Vitamin D-dependent chromatin association of CTCF in human monocytes. Biochim. Biophys. Acta 1859, 13801388. doi: 10.1016/j.bbagrm.2016.08.008

Neme, A., Seuter, S., and Carlberg, C. (2017). Selective regulation of biological processes by vitamin $\mathrm{D}$ based on the spatio-temporal cistrome of its receptor. Biochim. Biophys. Acta 1860, 952-961. doi: 10.1016/j.bbagrm.2017. 07.002

Neme, A., Seuter, S., Malinen, M., Nurmi, T., Tuomainen, T. P., Virtanen, J. K., et al. (2019). In vivo transcriptome changes of human white blood cells in response to vitamin D. J. Steroid Biochem. Mol. Biol. doi: 10.1016/j.jsbmb.2018.11.019 [Epub ahead of print].

Nurminen, V., Neme, A., Ryynanen, J., Heikkinen, S., Seuter, S., and Carlberg, C. (2015). The transcriptional regulator BCL6 participates in the secondary gene regulatory response to vitamin D. Biochim. Biophys. Acta 1849, 300-308. doi: 10.1016/j.bbagrm.2014.12.001

Nurminen, V., Neme, A., Seuter, S., and Carlberg, C. (2018). The impact of the vitamin D-modulated epigenome on VDR target gene regulation. Biochim. Biophys. Acta 1861, 697-705. doi: 10.1016/j.bbagrm.2018. 05.006

Nurminen, V., Neme, A., Seuter, S., and Carlberg, C. (2019). Modulation of vitamin D signaling by the pioneer factor CEBPA. Biochim. Biophys. Acta 1862, 96-106. doi: 10.1016/j.bbagrm.2018.12.004

Pereira, F., Barbachano, A., Silva, J., Bonilla, F., Campbell, M. J., Munoz, A., et al. (2011). DM6B/JMJD3 histone demethylase is induced by vitamin D and modulates its effects in colon cancer cells. Hum. Mol. Genet. 20, 4655-4665. doi: $10.1093 / \mathrm{hmg} / \mathrm{ddr} 399$

Polly, P., Herdick, M., Moehren, U., Baniahmad, A., Heinzel, T., and Carlberg, C. (2000). VDR-Alien: a novel, DNA-selective vitamin D3 receptorcorepressor partnership. FASEB J. 14, 1455-1463. doi: 10.1096/fasebj.14. 10.1455

Ramagopalan, S. V., Heger, A., Berlanga, A. J., Maugeri, N. J., Lincoln, M. R., Burrell, A., et al. (2010). A ChIP-seq defined genome-wide map of vitamin D receptor binding: associations with disease and evolution. Genome Res. 20, 1352-1360. doi: 10.1101/gr.107920.110
Reeves, R. K., Takahashi, H., Hatta, Y., Iriyama, N., Hasegawa, Y., Uchida, H., et al. (2014). Induced differentiation of human myeloid leukemia cells into M2 macrophages by combined treatment with retinoic acid and $1 \alpha, 25-$ dihydroxyvitamin D3. PLoS One 9:e113722. doi: 10.1371/journal.pone.011 3722

Rook, G. A., Steele, J., Fraher, L., Barker, S., Karmali, R., O’Riordan, J., et al. (1986). Vitamin D3, gamma interferon, and control of proliferation of Mycobacterium tuberculosis by human monocytes. Immunology 57, 159-163.

Seuter, S., Heikkinen, S., and Carlberg, C. (2013a). Chromatin acetylation at transcription start sites and vitamin $\mathrm{D}$ receptor binding regions relates to effects of 1 1 ,25-dihydroxyvitamin D3 and histone deacetylase inhibitors on gene expression. Nucleic Acids Res. 41, 110-124. doi: 10.1093/nar/ gks959

Seuter, S., Neme, A., and Carlberg, C. (2014a). Characterization of genomic vitamin $\mathrm{D}$ receptor binding sites through chromatin looping and opening. PLoS One 9:e96184. doi: 10.1371/journal.pone.0096184

Seuter, S., Neme, A., and Carlberg, C. (2016). Epigenome-wide effects of vitamin $\mathrm{D}$ and their impact on the transcriptome of human monocytes involve CTCF. Nucleic Acids Res. 44, 4090-4104. doi: 10.1093/nar/ gkv1519

Seuter, S., Neme, A., and Carlberg, C. (2017a). Epigenomic PU.1-VDR crosstalk modulates vitamin D signaling. Biochim. Biophys. Acta 1860, 405-415. doi: 10.1016/j.bbagrm.2017.02.005

Seuter, S., Neme, A., and Carlberg, C. (2018). ETS transcription factor family member GABPA contributes to vitamin D receptor target gene regulation. J. Steroid Biochem. Mol. Biol. 177, 46-52. doi: 10.1016/j.jsbmb.2017. 08.006

Seuter, S., Pehkonen, P., Heikkinen, S., and Carlberg, C. (2013b). Dynamics of $1 \alpha, 25$-dihydroxyvitamin D-dependent chromatin accessibility of early vitamin D receptor target genes. Biochim. Biophys. Acta 1829, 1266-1275. doi: 10.1016/ j.bbagrm.2013.10.003

Seuter, S., Pehkonen, P., Heikkinen, S., and Carlberg, C. (2013c). The gene for the transcription factor BHLHE40/DEC1/stra13 is a dynamically regulated primary target of the vitamin D receptor. J. Steroid Biochem. Mol. Biol. 136, 62-67. doi: 10.1016/j.jsbmb.2012.11.011

Seuter, S., Ryynänen, J., and Carlberg, C. (2014b). The ASAP2 gene is a primary target of 1,25-dihydroxyvitamin D in human monocytes and macrophages. J. Steroid Biochem. Mol. Biol. 144, 12-18. doi: 10.1016/j.jsbmb.2013. 08.014

Seuter, S., Virtanen, J. K., Nurmi, T., Pihlajamäki, J., Mursu, J., Voutilainen, S., et al. (2017b). Molecular evaluation of vitamin D responsiveness of healthy young adults. J. Steroid Biochem. Mol. Biol. 174, 314-321. doi: 10.1016/j.jsbmb.2016. 06.003

The Encode-Project-Consortium, Bernstein, B. E., Birney, E., Dunham, I., Green, E. D., Gunter, C., et al. (2012). An integrated encyclopedia of DNA elements in the human genome. Nature 489, 57-74. doi: 10.1038/nature 11247

Tsuchiya, S., Yamabe, M., Yamaguchi, Y., Kobayashi, Y., Konno, T., and Tada, K. (1980). Establishment and characterization of a human acute monocytic leukemia cell line (THP-1). Int. J. Cancer 26, 171-176. doi: 10.1002/ijc. 2910260208

Tuoresmäki, P., Väisänen, S., Neme, A., Heikkinen, S., and Carlberg, C. (2014). Patterns of genome-wide VDR locations. PLoS One 9:e96105. doi: 10.1371/ journal.pone.0096105

Verma, R., Jung, J. H., and Kim, J. Y. (2014). 1,25-Dihydroxyvitamin D3 upregulates TLR10 while down-regulating TLR2, 4, and 5 in human monocyte THP-1. J. Steroid Biochem. Mol. Biol. 141, 1-6. doi: 10.1016/j.jsbmb.2013. 12.012

Verway, M., Bouttier, M., Wang, T. T., Carrier, M., Calderon, M., An, B. S., et al. (2013). Vitamin D induces interleukin-lbeta expression: paracrine macrophage epithelial signaling controls $M$. tuberculosis infection. PLoS Pathog. 9:e1003407. doi: 10.1371/journal.ppat. 1003407

Vukic, M., Neme, A., Seuter, S., Saksa, N., de Mello, V. D., Nurmi, T., et al. (2015). Relevance of vitamin D receptor target genes for monitoring the vitamin D responsiveness of primary human cells. PLoS One 10:e0124339. doi: 10.1371/ journal.pone.0124339 
Wei, Z., Yoshihara, E., He, N., Hah, N., Fan, W., Pinto, A. F. M., et al. (2018). Vitamin D switches BAF complexes to protect beta cells. Cell 173, 11351149.e15. doi: 10.1016/j.cell.2018.04.013

Wilfinger, J., Seuter, S., Tuomainen, T.-P., Virtanen, J. K., Voutilainen, S., Nurmi, T., et al. (2014). Primary vitamin D receptor target genes as biomarkers for the vitamin D3 status in the hematopoietic system. J. Nutr. Biochem. 25, 875-884. doi: 10.1016/j.jnutbio.2014.04.002

Wu, S., Ren, S., Nguyen, L., Adams, J. S., and Hewison, M. (2007). Splice variants of the CYP27b1 gene and the regulation of 1,25-dihydroxyvitamin D3 production. Endocrinology 148, 3410-3418. doi: 10.1210/en.2006-1388
Conflict of Interest Statement: The authors declare that the research was conducted in the absence of any commercial or financial relationships that could be construed as a potential conflict of interest.

Copyright ( 2019 Nurminen, Seuter and Carlberg. This is an open-access article distributed under the terms of the Creative Commons Attribution License (CC BY). The use, distribution or reproduction in other forums is permitted, provided the original author(s) and the copyright owner(s) are credited and that the original publication in this journal is cited, in accordance with accepted academic practice. No use, distribution or reproduction is permitted which does not comply with these terms. 